\title{
Vitamin D Status in Neonates with Congenital Heart Disease: Its Changes after Heart Surgery and Relationship to the Outcome
}

\author{
Ziba Mosayebi1 $^{1}$, Mohamadreza Mirzaaghayan1(D), Aliakbar Zeinaloo ${ }^{1}(\mathbb{D})$ \\ Behdad Gharib $^{1}$ (D), Pedram Ghahremani2*iD
}

1. Children's Medical Center, Tehran University of Medical Sciences, Tehran, Iran

2. Dept. of Pediatrics, Mousavi Hospital, School of Medicine, Zanjan University of Medical Sciences, Zanjan, Iran

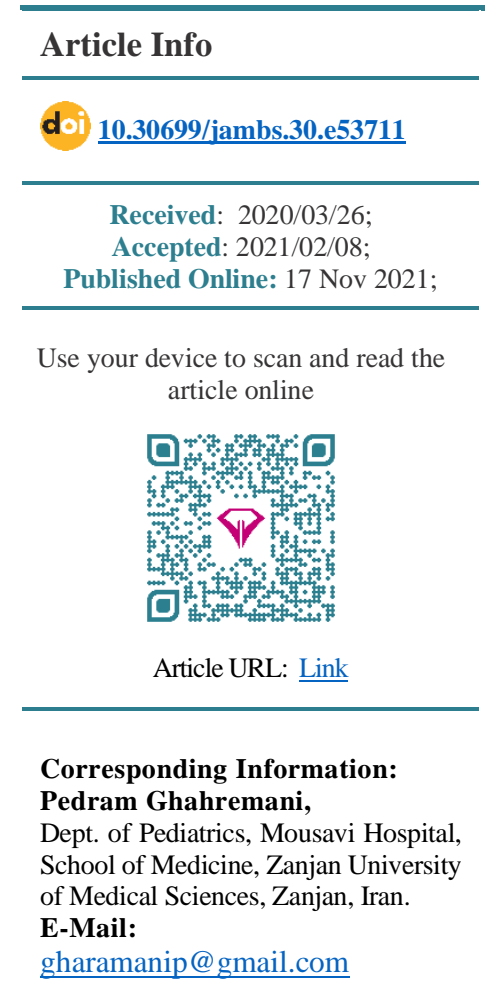

\section{ABSTRACT}

Background \& Objective: Vitamin D deficiency has been implicated in a wide range of conditions such as infectious and autoimmune diseases, cardiovascular and cerebrovascular events, and various malignancies to unfavorable birth outcomes. We studied vitamin D status in a group of neonates with congenital heart disease (CHD) and its relationship with the outcome of heart surgery.

Materials \& Methods: Total vitamin D levels in plasma were measured before and 24 hours after the operation in 45 neonates with CHD undergoing heart surgery and in 77 hospitalized neonates without a history of CHD as controls, at Children's Medical Center between March and September 2018.

Results: Preoperative vitamin D levels in patients with CHD were not significantly different from the control group $(42.4+/-18.0$ versus $46.9+/-27.7 \mathrm{nmol} / \mathrm{L}, \mathrm{P}=0.640)$. Patients' vitamin D levels decreased postoperatively $(42.4+/-18.0$ versus $36.2+$ +/$14.5 \mathrm{nmol} / \mathrm{L}, \mathrm{P}=0.013)$. This decline was significant in the cyanotic and open-heart surgery groups, but not in non-cyanotic or closed-heart surgery groups. In 41 (91.1\%) patients, the outcome was successful discharge from the hospital, in $3(6.7 \%)$, the outcome was demise, and in one, it was not determined due to early discharge. Preoperative and postoperative vitamin D levels did not show any relationship with the outcome. However, logistic regression analysis revealed a significant relationship between the amount of postoperative decline in vitamin $\mathrm{D}$ levels and the outcome of death $(\mathrm{OR}=1.261,95 \% \mathrm{CI}=1.026-1.551, \mathrm{P}=0.028)$.

Conclusion: Results corroborates previous findings and suggests the amount of postoperative decline in vitamin $\mathrm{D}$ levels as a predictor of the outcome of heart surgery in CHD.

Keywords: Congenital Heart Defects, Vitamin D, Newborn

Copyright $\odot$ 2022, This is an original open-access article distributed under the terms of the Creative Commons Attribution-noncommercial 4.0 International License which permits copy and redistribution of the material just in noncommercial usages with proper citation.

\section{Introduction}

Classical roles of vitamin D in intestinal calcium absorption and renal calcium reabsorption and its dynamic skeletal effects are well-established, as are the consequences of its deficiency in terms of skeletal abnormalities such as rickets $(\mathbf{1}, 2)$. Furthermore, in recent years, vitamin $\mathrm{D}$ deficiency has been implicated in a wide range of conditions from infectious and autoimmune diseases, cardiovascular and cerebrovascular events, and various malignancies, to unfavorable birth outcomes and increased all-cause mortality (3-8). Thus, vitamin D has been recognized as a pleiotropic factor with diverse functions that are still under study.

One interesting area of recent focus has been vitamin D status and shifts in vitamin D levels in critically ill patients and these variables' relationship to the outcome. In view of the varied roles of vitamin D in essential organs and life processes, it has been postulated that deficiency in this vitamin may adversely affect the outcome in critically ill patients (9). Several studies have found an association between vitamin $\mathrm{D}$ deficiency and the risk of death in adults in critical care (10-12), and clinical trials are being performed to investigate the possible beneficial role of supplemental vitamin D in this group of patients (13-16). Furthermore, a growing number of studies have addressed the role of vitamin D in critically ill pediatric patients (1723). These studies have found mean vitamin $D$ levels in critically ill children to be lower compared to control subjects and have established associations between vitamin D deficiency and increased risk of mortality, disease severity, and complications in these patients (24).

In recent years, several studies have focused on vitamin D status in pediatric patients with congenital heart disease 
(CHD) undergoing heart surgery and its relationship to the outcome. In 2013, McNally et al. reported results of a longitudinal study of vitamin D status of 54 patients with CHD with a mean age of 8.4 (from 2.4 to 66.5) months who underwent open-heart surgery and 4 children with coarctation of aorta who underwent closed-heart surgery. Patients' vitamin D levels declined significantly after the operation, and this decrease was more pronounced in the open-heart surgery group. Almost all the decline in this group occurred during the operation (25).

In other studies, Graham et al. reported the vitamin D status in 70 neonates with CHD undergoing open-heart surgery in 2013 (26). No significant decrease was observed in vitamin D levels after the operation. However, a negative correlation was found between vitamin D levels after surgery and the need for inotropes. In 2017, Abou Zahr et al. reported an investigation of vitamin D levels in 20 patients with $\mathrm{CHD}$ undergoing open-heart surgery. Subjects ranged from 2 months to 17 years of age (27). The authors measured vitamin D levels before and immediately after surgery and detected a significant decline in the mean vitamin D level after the operation. In 2020, Dohain et al. studied vitamin D status in 69 children with CHD undergoing open-heart surgery (28). They found vitamin D deficiency in $49.3 \%$ of the patients. This figure increased to $91.3 \%$ postoperatively.

In the present paper, we report vitamin D status and changes in vitamin D levels after the operation in a group of neonates with CHD undergoing heart surgery at Children's Medical Center of Tehran University of Medical Sciences and the relationship of these vitamin D variables with the outcomes of demise or successful discharge from hospital.

\section{Materials and Methods}

Study and control groups: The study group comprised neonates with CHD who underwent heart surgery at Children's Medical Center of Tehran University of Medical Sciences between March and September of 2018. The diagnosis of CHD was verified through echocardiography by a pediatric cardiologist. Subjects with a history of perinatal asphyxia or cardiopulmonary resuscitation (CPR) and preterm infants with gestational ages below 34 weeks were excluded from the study. Details on sex, gestational age, the specific variety of $\mathrm{CHD}$, age on the day of surgery, and outcome (demise versus successful discharge from hospital), were transcribed from patients' records. The control group was drawn from neonates without a history of CHD, hospitalized at the neonatal ward and NICU of the hospital during the same period.

Ethical standards: This study complies with the ethical standards of the relevant national guidelines and with the Helsinki Declaration of 1975, as revised in 2008, and has been approved by the institutional committee at Tehran University of Medical Sciences (Approval ID: IR.TUMS.CHMC.REC.1397.090). Informed consent was obtained from parents, and pertinent data were gathered from patients' records with due attention to confidentiality. Patients were identified by codes, and no personally identifiable information was recorded. Measurements of vitamin D levels were performed on blood samples taken during routine laboratory studies and entailed no additional blood-drawing.

Vitamin D measurements: Total vitamin D plasma levels in control and study groups were measured through the quantitative luminescence method. In subjects with CHD, vitamin D levels were measured immediately before and 24 hours after the operation. Levels below $49.9 \mathrm{nmol} / \mathrm{L}(20 \mathrm{ng} / \mathrm{mL})$ were designated as vitamin D deficiency (29).

Data analysis: Data were analyzed using SPSS software. Relationships between variables were examined using Mann-Whitney $U$ test, Wilcoxon signed-rank test, Fisher's exact test, McNemar's test, Spearman's rank correlation coefficient, Rank-Biserial Correlation, Phi coefficient, Linear regression, and Logistic regression as applicable. The significance level was set at 0.05 .

\section{Results}

Characteristics of the group under study: A total of 45 neonates with CHD were studied. Moreover, vitamin D levels were measured in 77 non-CHD neonates as the control group. Patients were categorized into cyanotic and non-cyanotic according to the characteristics of specific congenital heart diseases. Of the patients, $33(73.3 \%)$ had the cyanotic type of the disease, and $12(26.6 \%)$ were of the noncyanotic type. Transposition of the great arteries (TGA), in isolation or combined with other defects, was the most prevalent disorder with a frequency of $53.3 \%$ (Table 1). Of the patients, 32 (71.7\%) underwent open-heart surgery, and 13 (28.9\%) closedheart surgery. An outcome of survival and successful discharge from the hospital was recorded in $41(91.1 \%)$ of the patients. In 3 patients $(6.7 \%)$, the outcome was demise. In one case, the outcome could not be determined due to the early discharge of the patient at the parents' request. 
Table 1: CHD Types (Cyanotic or Non-cyanotic) and Specific Disease Varieties

\begin{tabular}{|ccc|}
\hline CHD Type and Variety & Number of Patients & Percent \\
\hline Cyanotic & $(33)$ & $(73.3)$ \\
TGA & 21 & 46.6 \\
\hline TGA+VSD & 2 & 4.4 \\
\hline TGA+VSAD+PA & 1 & 2.2 \\
\hline TGA+PDA & 1 & 2.2 \\
\hline TAPVC & 3 & 6.6 \\
\hline TA & 1 & 2.2 \\
\hline PA/IVS+ASD+PDA & 1 & 2.2 \\
\hline PA+VSD+PDA & 1 & 2.2 \\
\hline DORV+PS+TOF & 1 & 2.2 \\
\hline DORV+CoA+VSD & 1 & 2.2 \\
\hline Non-cyanotic & $(12)$ & $(26.6)$ \\
\hline CoA & 5 & 11 \\
\hline CoA+VSD & 2 & 4.4 \\
\hline CoA+VSD+PDA & 1 & 2.2 \\
\hline PDA & 3 & 6.6 \\
\hline TAA & 1 & 2.2 \\
\hline
\end{tabular}

$\mathrm{ASD}=$ Atrial septal defect, $\mathrm{CoA}=$ Coarctation of the aorta, $\mathrm{DORV}=$ Double outlet right ventricle, IAA $=$ Interrupted aortic arch, $\mathrm{PA}=$ Pulmonary atresia, PA/IVS= Pulmonary atresia with intact ventricular septum, PDA= Patent ductus arteriosus, PS= Pulmonary stenosis, $\mathrm{TA}=$ Tricuspid atresia, $\mathrm{TAPVC}=$ Total anomalous pulmonary venous connection, $\mathrm{TGA}=$ Transposition of the great arteries, $\mathrm{TOF}=$ Tetralogy of Fallot, VSD $=$ Ventricular septal defect

Vitamin D status before and after operation: Before the operation, vitamin D levels in patients with CHD were not significantly different from that of the control group $(42.4+/-18.0 \mathrm{nmol} / \mathrm{L}$ versus $46.9+/-27.7 \mathrm{nmol} / \mathrm{L}$, $\mathrm{P}=0.640)$. With heart surgery, vitamin $\mathrm{D}$ levels in patients with CHD decreased significantly $(42.4+/-18.0 \mathrm{nmol} / \mathrm{L}$ before the operation versus $36.2+/-14.5 \mathrm{nmol} / \mathrm{L}$ after it, $\mathrm{P}=0.013$ ), and the difference between CHD and control groups became significant $(46.9+/-27.7 \mathrm{nmol} / \mathrm{L}$ versus $36.2+/-14.5 \mathrm{nmol} / \mathrm{L}, \mathrm{P}=0.041)$. Furthermore, the proportion of vitamin D deficient patients in the CHD group increased significantly after the surgery $(66.7 \%$ of the patients before surgery versus $84.4 \%$ after it, $\mathrm{P}=0.021$ ) and became significantly higher than the control group (84.4\% versus $60.3 \%, \mathrm{P}=0.008$ ).

Both before and after the operation, vitamin D levels in the non-cyanotic group were significantly lower compared to the cyanotic group, $(34.4+/-17.7 \mathrm{nmol} / \mathrm{L}$ versus $45.4+/-17.2 \mathrm{nmol} / \mathrm{L}, \mathrm{P}=0.039$ before the operation and $30.7+/-16.0 \mathrm{nmol} / \mathrm{L} 38.4+/-13.5 \mathrm{nmol} / \mathrm{L}, \mathrm{P}=0.017$ after the operation).

In the cyanotic group, vitamin $\mathrm{D}$ levels declined significantly after the operation $(45.4+/-17.2 \mathrm{nmol} / \mathrm{L}$ versus $38.4+/-13.5 \mathrm{nmol} / \mathrm{L}, \mathrm{P}=0.033)$, and the proportion of vitamin $\mathrm{D}$ deficient patients increased significantly
(60\% versus $82 \%, \mathrm{P}=0.039)$, but in the non-cyanotic group, changes in these variables after the operation did not reach significant levels. Similarly, in the open-heart surgery group, vitamin D levels declined significantly after the operation $(43.4+/-16.7 \mathrm{nmol} / \mathrm{L}$ versus $37.2+/$ $14.0 \mathrm{nmol} / \mathrm{L}, \mathrm{P}=0.045)$, and the proportion of vitamin $\mathrm{D}$ deficient patients increased ( $66 \%$ versus $84 \%, \mathrm{P}=0.034$ ). No significant changes were observed in these variables in the closed-surgery group after the operation.

Vitamin D status in relation to the outcome: In the group with an outcome of demise, vitamin D levels decreased from $57.4+/-34.3 \mathrm{nmol} / \mathrm{L}$ before the operation to $29.1+/-11.8 \mathrm{nmol} / \mathrm{L}$ after it, while in the group with the outcome of survival, the level of vitamin $\mathrm{D}$ was 41.3 $+/-16.4 \mathrm{nmol} / \mathrm{L}$ before the operation and decreased to $36.8+/-14.8 \mathrm{nmol} / \mathrm{L}$ after it. All patients with an outcome of death were of the cyanotic type, though of different disease varieties, and all underwent open-heart surgery. Both in the group of cyanotic patients with an outcome of survival and the group of open surgery with an outcome of survival, the difference between vitamin $\mathrm{D}$ levels before and after the operation was not significant $(44.2+/$ $15.2 \mathrm{nmol} / \mathrm{L}$ before surgery versus $39.2+/-13.5 \mathrm{nmol} / \mathrm{L}$ after surgery $\mathrm{P}=0.092$ for the group of cyanotic patients with an outcome of survival; and $43.5+/-16.6 \mathrm{nmol} / \mathrm{L}$ 
before surgery versus $37.3+/-13.9 \mathrm{nmol} / \mathrm{L}$ after surgery $\mathrm{P}=0.126$ for the group of open-heart surgery patients with an outcome of survival).

Levels of vitamin D in the patients, before or after the operation, did not show any relationship with the outcome. However, logistic regression revealed a significant relationship between the amount of postoperative decline in vitamin $\mathrm{D}$ levels and the outcome of death $(\mathrm{OR}=1.261,95 \% \mathrm{CI}=1.026-1.551, \mathrm{P}=0.028)$. The results of this analysis are summarized in table 2 .

Table 2. Logistic Regression Analysis of Risk of Death in CHD Neonates Undergoing Heart Surgery

\begin{tabular}{ccccccc} 
& Coeff. & Standard Error & $\mathrm{p}$ Value & Odds Ratio & CI Lower Limit & CI Upper Limit \\
\hline Vi. D Decline & .232 & .105 & .028 & 1.261 & 1.026 & 1.551 \\
Constant & -4.070 & 1.237 & .001 & .017 & & \\
\hline
\end{tabular}

Vit. D Decline= postoperative decline in vitamin D level; Coeff.= coefficient expressed in logits; CI $=95 \%$ confidence interval for the odds ratio.

\section{Discussion:}

In the present study, we investigated vitamin D status and its changes in neonates with CHD undergoing heart surgery at Children's Medical Center of Tehran University of Medical Sciences. The relationship between these factors and the type of the disease (noncyanotic versus cyanotic), type of the surgery (closedheart versus open-heart surgery), and outcome (successful discharge versus demise), were studied. In recent years, several studies have addressed the question of vitamin D levels in patients with CHD, its changes during and after heart surgery, the possible relationship between these variables and the outcome of the operation, and the potential of vitamin D supplementation as a protective factor against unfavorable outcomes in this group of patients (25-27, 30).

The present study comprised a range of cyanotic and non-cyanotic congenital heart diseases and both openheart and closed-heart surgical procedures. This broad coverage provided the opportunity for analyzing and comparing cyanotic and non-cyanotic types of the disease, as well as open-heart and closed-heart surgical procedures, in relation to vitamin D status. Among previous studies, the study by McNally et al. comprised 54 open-heart surgery patients and 4 cases with closedheart surgery, and in the studies by Abou Zahr et al. and Graham et al., all patients underwent open-heart surgery (25-27). In terms of the outcome, in the present study $41(91 \%)$ cases improved after surgery and were successfully discharged while in $3(7 \%)$ subjects, the outcome was demise. One patient had an early discharge at the request of the parents. Previous studies did not report any patients with an outcome of demise.

Before the operation, vitamin D levels were not significantly different in CHD and control groups. This finding is in keeping with the findings of McNally et al., which reported that vitamin D levels in children with CHD before heart surgery were only slightly lower than normal Canadian children (25). The mean vitamin $\mathrm{D}$ level in patients with $\mathrm{CHD}$ decreased significantly after the operation and became significantly lower than that of the control group. Similarly, both McNally et al. and Abou Zahr et al. have previously reported a significant decrease in vitamin D levels after the operation $(25,27)$. By contrast, Graham et al. did not find such a decline, however, it should be noted that $84 \%$ of the patients in their study were already vitamin D-deficient before surgery (26).

After surgery, vitamin D levels decreased significantly in cyanotic patients but the decrease in non-cyanotic patients did not reach a significant level. Similarly, the proportion of the patients with vitamin D deficiency increased significantly in the cyanotic group after surgery but no significant changes were detected in the group of non-cyanotic patients.

After surgery, vitamin D levels decreased and the proportion of the patients with vitamin D deficiency increased significantly in the open-heart surgery, but no significant changes were detected in the group of closed-heart surgery. As mentioned, previous studies comprised mostly of open-heart surgery patients with CHD. Graham et al. did not report a significant decrease in the level of vitamin D after open-heart surgery (26), while McNally et al. and Abou Zahr et al. reported such a decline $(25,27)$. The study by McNally et al. included a number of closed-heart surgery patients (4 out of 58 subjects). In that study, the decline in the level of vitamin D after the operation was significantly greater in the group of open-heart surgery compared to the closed-heart surgery group (25). The findings of the present study are consistent with those results.

Preoperative level of vitamin D in infants with CHD did not show a correlation with the outcome after surgery; neither did it predict the rate of postoperative decline in vitamin D level, but interestingly, logistic regression analysis revealed a significant relationship between the rate of postoperative decline in vitamin $D$ levels and the outcome. Thus, a sharp postoperative decline in vitamin D levels is suggested as a predictor of the outcome of death. 
This is the first time that the relationship between changes in vitamin D levels during surgery and the outcome of death is studied in patients with CHD. In previous studies, there were no subjects with the outcome of death, and thus, there are no previous studies for comparison in this regard. However, it is noted that McNally et al. investigated the catecholamine requirement in patients with CHD undergoing surgery as an indicator of cardiovascular dysfunction. They reported an association between lower postoperative, but not preoperative, vitamin D levels and catecholamine requirement (25). Similarly, in the study by Graham et al., a lower vitamin D level after surgery, but not before it, was associated with an increased inotrope requirement (26). Likewise, Dohain et al. reported an association between lower postoperative levels of vitamin D and increased need for inotropic support (28). Thus, similar to our findings, these results point to a relationship between the postoperative decrease in vitamin D levels and unfavorable conditions.

It should be noted that the present study used convenience sampling and included cases and control subjects hospitalized at Children's Medical Center between March and September of 2018. This limitation of the study could potentially affect the power of the statistical analyses. Specifically, the lack of statistical significance in analyses involving the noncyanotic group might be due to the relatively small number of patients in this group.

\section{Conclusion:}

In conclusion, the results of the present study corroborate the findings of previous studies on vitamin D changes in infants with CHD undergoing heart surgery. Our findings further suggest that the rate of postoperative decline in vitamin $\mathrm{D}$ levels is a predictor of the outcome of surgery in infants with CHD. The results of the present study, along with similar studies, can contribute to a better understanding, prediction, and possible prevention of unfavorable outcomes in these patients. Further studies are needed to gain a more accurate picture of the significance of vitamin D change in this group of patients.

\section{Acknowledgment:}

The authors assert that there is no conflict of interest to declare and there has been no financial support.

\section{Conflicts of Interest:}

There is no conflict of interest.

\section{References}

1. Christakos S, Dhawan P, Verstuyf A, Verlinden L, Carmeliet G. Vitamin D: Metabolism, Molecular Mechanism of Action, and Pleiotropic Effects. Physiol Rev. 2016;96(1):365-408. [DOI:10.1152/physrev.00014.2015] [PMID] [PMCID]

2. McNally JD, Amrein K. Vitamin D Deficiency in Pediatric Critical Care. J Pediatr Intensive Care. 2016;5(4):142-53. [DOI:10.1055/s-00361583285] [PMID] [PMCID]

3. Caprio M, Infante M, Calanchini M, Mammi C, Fabbri A. Vitamin D: not just the bone. Evidence for beneficial pleiotropic extraskeletal effects. Eat Weight Disord. 2017;22(1):27-41. [DOI:10.1007/s40519-016-0312-6] [PMID]

4. Gaksch M, Jorde R, Grimnes G, Joakimsen R, Schirmer H, Wilsgaard T, et al. Vitamin D and mortality: Individual participant data metaanalysis of standardized 25-hydroxyvitamin D in 26916 individuals from a European consortium. PloS one. 2017;12(2):e0170791.

5. Hewison M, Wagner CL, Hollis BW. Vitamin D Supplementation in Pregnancy and Lactation and Infant Growth. N Engl J Med. 2018;379(19):18801. [DOI:10.1056/NEJMc1812157]

6. Pludowski P, Grant WB, Konstantynowicz J, Holick MF. Editorial: Classic and Pleiotropic Actions of Vitamin D. Front Endocrinol. 2019;10:341. [DOI:10.3389/fendo.2019.00341] [PMID] [PMCID]

7. Pludowski P, Holick MF, Pilz S, Wagner CL, Hollis BW, Grant WB, et al. Vitamin D effects on musculoskeletal health, immunity, autoimmunity, cardiovascular disease, cancer, fertility, pregnancy, dementia and mortality-a review of recent evidence. Autoimmun Rev. 2013;12(10):976-89. [DOI:10.1016/j.autrev.2013.02.004] [PMID]

8. Souberbielle JC, Body JJ, Lappe JM, Plebani M, Shoenfeld Y, Wang TJ, et al. Vitamin D and musculoskeletal health, cardiovascular disease, autoimmunity and cancer: Recommendations for clinical practice. Autoimmun Rev. 2010;9(11):709-15.

[DOI:10.1016/j.autrev.2010.06.009] [PMID]

9. Lee P, Nair P, Eisman JA, Center JR. Vitamin D deficiency in the intensive care unit: an invisible accomplice to morbidity and mortality? Intensive Care Med. 2009;35(12):2028-32. [DOI:10.1007/s00134-009-1642-x] [PMID] 
10. de Haan K, Groeneveld AB, de Geus HR, Egal M, Struijs A. Vitamin D deficiency as a risk factor for infection, sepsis and mortality in the critically ill: systematic review and meta-analysis. Crit Care. 2014;18(6):660. [DOI:10.1186/s13054-014-06604] [PMID] [PMCID]

11. Zajic P, Amrein K. Vitamin D deficiency in the ICU: a systematic review. Minerva Endocrinol. 2014;39(4):275-87.

12. Zhang YP, Wan YD, Sun TW, Kan QC, Wang LX. Association between vitamin D deficiency and mortality in critically ill adult patients: a metaanalysis of cohort studies. Crit Care. 2014;18(6):684. [DOI:10.1186/s13054-014-06849] [PMID] [PMCID]

13. Amrein K, Schnedl C, Holl A, Riedl R, Christopher KB, Pachler C, et al. Effect of highdose vitamin D3 on hospital length of stay in critically ill patients with vitamin D deficiency: the VITdAL-ICU randomized clinical trial. Jama. 2014;312(15):1520-30.

\section{[DOI:10.1001/jama.2014.13204] [PMID]}

14. Amrein K, Sourij H, Wagner G, Holl A, Pieber TR, Smolle KH, et al. Short-term effects of high-dose oral vitamin D3 in critically ill vitamin D deficient patients: a randomized, double-blind, placebocontrolled pilot study. Crit Care. 2011;15(2):R104. [DOI:10.1186/cc10120] [PMID] [PMCID]

15. Han JE, Jones JL, Tangpricha V, Brown MA, Brown LAS, Hao L, et al. High Dose Vitamin D Administration in Ventilated Intensive Care Unit Patients: A Pilot Double Blind Randomized Controlled Trial. J Clin Transl Endocrinol. 2016;4:59-65. [DOI:10.1016/i.jcte.2016.04.004] [PMID] [PMCID]

16. Nair P, Venkatesh B, Lee P, Kerr S, Hoechter DJ, Dimeski G, et al. A Randomized Study of a Single Dose of Intramuscular Cholecalciferol in Critically Ill Adults. Crit Care Med. 2015;43(11):2313-20. [DOI:10.1097/CCM.0000000000001201] [PMID]

17. Madden K, Feldman HA, Smith EM, Gordon CM, Keisling SM, Sullivan RM, et al. Vitamin D deficiency in critically ill children. Pediatrics. 2012;130(3):421-8. [DOI:10.1542/peds.20113328] [PMID] [PMCID]

18. Rippel C, South M, Butt WW, Shekerdemian LS. Vitamin D status in critically ill children. Intensive Care Med. 2012;38(12):2055-62. [DOI:10.1007/s00134-012-2718-6] [PMID]

19. Ayulo M, Jr., Katyal C, Agarwal C, Sweberg T, Rastogi D, Markowitz M, et al. The prevalence of vitamin $\mathrm{D}$ deficiency and its relationship with disease severity in an urban pediatric critical care unit. Endocr Regul. 2014;48(2):69-76. [DOI:10.4149/endo_2014_02_69] [PMID]
20. Hebbar KB, Wittkamp M, Alvarez JA, McCracken CE, Tangpricha V. Vitamin D Deficiency in Pediatric Critical Illness. J Clin Transl Endocrinol. 2014;1(4):170-5.

[DOI:10.1016/j.jcte.2014.09.002] [PMCID]

21. McNally JD, Menon K, Chakraborty P, Fisher L, Williams KA, Al-Dirbashi OY, et al. The association of vitamin D status with pediatric critical illness. Pediatrics. 2012;130(3):429-36. [DOI:10.1542/peds.2011-3059] [PMID]

22. McNally JD, Menon K, Lawson ML, Williams K, Doherty DR. 1,25-Dihydroxyvitamin D Levels in Pediatric Intensive Care Units: Risk Factors and Association With Clinical Course. J Clin Endocrinol Metab. 2015;100(8):2942-5. [DOI:10.1210/jc.2014-4471] [PMID]

23. Onwuneme C, Carroll A, Doherty D, Bruell H, Segurado R, Kilbane M, et al. Inadequate vitamin $\mathrm{D}$ levels are associated with culture positive sepsis and poor outcomes in paediatric intensive care. Acta Paediatr. 2015;104(10):e433-8. [DOI:10.1111/apa.13090] [PMID]

24. McNally JD, Nama N, O'Hearn K, Sampson M, Amrein K, Iliriani K, et al. Vitamin D deficiency in critically ill children: a systematic review and meta-analysis. Crit Care. 2017;21(1):287. [DOI:10.1186/s13054-017-1875-y] [PMID] [PMCID]

25. McNally JD, Menon K, Chakraborty P, Fisher L, Williams KA, Al-Dirbashi OY, et al. Impact of anesthesia and surgery fo congenital heart disease on the vitamin d status of infants and children: a prospective longitudinal study. Anesthesiology. 2013;119(1):71-80.

[DOI:10.1097/ALN.0b013e31828ce817] [PMID]

26. Graham EM, Taylor SN, Zyblewski SC, Wolf B, Bradley SM, Hollis BW, et al. Vitamin D status in neonates undergoing cardiac operations: relationship to cardiopulmonary bypass and association with outcomes. J Pediatr 2013;162(4):823-6.

[DOI:10.1016/j.jpeds.2012.10.013] [ [PMID] [PMCID]

27. Abou Zahr R, Faustino EVS, Carpenter $\mathrm{T}$, Kirshbom P, Hall EK, Fahey JT, et al. Vitamin D Status After Cardiopulmonary Bypass in Children With Congenital Heart Disease. J Intensive Care Med. 2017;32(8):508-13. [DOI:10.1177/0885066616652077] [PMID]

28. Dohain AM, Almogati J, Al-Radi OO, Elassal AA, Zaher ZF, Fatani TH, et al. Serum vitamin D status following pediatric cardiac surgery and association with clinical outcome. Eur J Pediatr 2020;179(4):635-43. [DOI:10.1007/s00431-01903538-x] [PMID] 
29. Wagner CL, Greer FR. Prevention of rickets and vitamin D deficiency in infants, children, and adolescents. Pediatrics. 2008;122(5):1142-52. [DOI:10.1542/peds.2008-1862] [PMID]
30. McNally JD, O'Hearn K, Lawson ML, Maharajh G, Geier P, Weiler H, et al. Prevention of vitamin $D$ deficiency in children following cardiac surgery: study protocol for a randomized controlled trial. Trials. 2015;16:402. [DOI:10.1186/s13063-0150922-8] [PMID] [PMCID]

\section{How to Cite This Article:}

Mosayebi Z, Mirzaaghayan M, Zeinaloo A, Gharib B, Ghahremani P. Vitamin D Status in Neonates with Congenital Heart Disease: Its Changes after Heart Surgery and Relationship to the Outcome. J Adv Med Biomed Res. 2022; 30 (138): 17-23

\section{Download citation:}

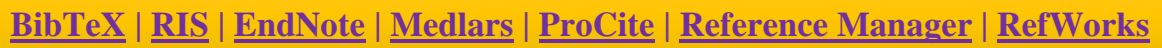

